\title{
Variación en la calidad del agua de riego en un huerto de cítricos ${ }^{1}$
}

\author{
Otávio A. de Almeida ${ }^{2} \&$ Juan M. Gisbert ${ }^{3}$
}

\begin{abstract}
RESUMEN
Con el objetivo de evaluar la variación en la calidad del agua de riego por goteo en un huerto de cítricos y sus consecuencias sobre el suelo y las plantas se realizó un ensayo en la finca "El Saladar", situada en la población de Sueca, Valencia, España. Durante toda la temporada del estudio se muestreó periódicamente las aguas de la A. R. del Júcar, del canal de conducción para el riego de arroz, y la utilizada para el riego de cítricos en la finca, en el sitio destinado a la captación, para determinar su calidad y su variación. También se hizo muestreo con la misma periodicidad del agua de drenaje, colectada en pozos de observaciones instalados en cada cuadrante del área del experimento. Considerando los resultados obtenidos, se puede concluir que la mezcla de las aguas de drenaje con el agua de la A. R. del Júcar, en general es clasificada como $\mathrm{C}_{3} \mathrm{~S}_{1}$ y, según las directrices de la $\mathrm{FAO}$, no presenta ningún grado de restricción de uso; Es posible la utilización de la mezcla de agua de buena calidad (P. ej. CE entre 1,18-1,43 dS m-1 ${ }^{-1}$ RAS y RAS ${ }^{\circ}$ entre 1,371,81 y $1,74-2,43\left(\mathrm{mmol}_{\mathrm{C}} \mathrm{L}^{-1}\right)^{1 / 2}$, con una agua de calidad dudosa (P. ej. CE entre 3,05-4,21 dS m $\mathrm{m}^{-1}$, RAS y RAS ${ }^{\circ}$ entre 2,83-4,00 y 3,41-4,84 $\left(\mathrm{mmol}_{\mathrm{C}} \mathrm{L}^{-1}\right)^{1 / 2}$, durante un largo periodo de tiempo, sin salinizar ni sodificar el suelo; La mezcla de agua utilizada en el riego de cítricos no influenció en el desarrollo de la planta.
\end{abstract}

Palabras claves: salinidad, sodicidad, suelos salinos, agua salina

\section{The variation in the water quality in an orchard of citrus}

\begin{abstract}
An experiment was carried out with the objective of evaluating the variation in the water quality in drip irrigation in an orchard of citrus and their consequences on the soil and plants at the farm "El Saladar", located in the population of "Sueca", Valencia, Spain. During the whole season of the study, the water of the A. R. of the Júcar used for citrus irrigation, were sampled from conveyance conduction channel for rice irrigation in order to determine water quality and its variation. Also the drainage water was sampled from observation wells installed in each quadrant of the area of the experiment. Considering the obtained results it can be concluded that the mixture of the drainage water with the water of the Real Canal of the Júcar is classified as $\mathrm{C}_{3} \mathrm{~S}_{1}$ and, according to the guidelines of the FAO it does not present any degree of use restriction. It is possible to utilize the mixture of good water (EC between 1.18-1.43 dS $\mathrm{m}^{-1}$, SAR and SAR ${ }^{\circ}$ between 1.37-1.81 and 1.74-2.43( $\left.\mathrm{mmol} \mathrm{L}^{-1}\right)^{1 / 2}$, with a doubtful quality water (EC between 3.05-4.21 $\mathrm{dS} \mathrm{m}^{-1}$, SAR and SAR between $2.83-4.00$ and 3.41-4.84 (mmol L-1 $)^{1 / 2}$, during a long time, without having a saline or sodic soil; the mixture of water used in the irrigation of citric fruits did not influence the development of the plant.
\end{abstract}

Key words: salinity, sodicity, saline soil, saline water

1 Trabalho extraído da Tese de Doutorado do primeiro autor, apresentada na Universidade Politécnica de Valencia, Espanha

${ }^{2}$ Embrapa Mandioca e Fruticultura Tropical. C.P. 007, CEP 44380-000, Cruz das Almas, BA. Fone: (0xx75) 3621.8046, Fax: (0xx75) 3621 8097. E-mail: otavio@cnpmf.embrapa.br. (Foto).

${ }_{3}^{3}$ Depto. de Producción Vegetal, UDSuelos/Universidad Politécnica Valencia, Camino de Vera 14. CEP. 46020 Valencia -España. Fone: 963877330 - Fax; 96 387 7339. E-mail: jgisbert@prv.upv.es 


\section{MATERIAL Y MÉTODOS}

\section{INTRODUCCIÓN}

La producción de cultivo en regiones áridas y semiáridas del mundo depende de abastecimiento adecuado de agua de calidad apropiada. En áreas de agricultura de regadío donde frecuentemente hay escasez de agua de riego, se pone énfasis comúnmente en métodos para aumentar la cantidad de agua, por ejemplo, utilizando agua de drenaje (Grattan \& Rhoades, 1990); o agua subterránea (Howitt \& M’Marete 1991), en lugar de considerar si las demandas de agua puestas sobre tales regiones áridas han sido demasiado altas para asegurar un abastecimiento a largo plazo fiable. En muchas regiones áridas de Australia, Egipto, India, Israel, Estados Unidos y Unión Soviética, es necesario el desarrollo de nuevos abastecimientos de agua de riego para mantener un abastecimiento alimentario estable. A fin de aumentar los recursos de agua, el país o la región debe ser capaz de utilizar aguas de mala calidad. Las posibles fuentes son las aguas que se pierden en los canales de las tierras regadas o de los tubos de drenaje y aguas que están presentes en sistemas de agua subterránea poco profundas. La estrategia de uso de estas aguas está en presuponer dos fuentes de agua: una de buena calidad (no salina) y otra de más pobre calidad (salina), aunque no es necesario que estén disponibles simultáneamente durante todo el año o toda la temporada

Diversos pesquisidores (Rhoades 1972, 1977, 1984; Meiri et al., 1986; Ayars et al., 1986a, b; Grattan et al., 1987; Grattan \& Rhoades, 1990, Grattan, 1994; Shennan et al., 1987; Rhoades et al., 1988, 1989), llegaron a la conclusión de que el uso de agua salina para el riego es factible, especialmente cuando las aguas se alternan o se mezclan con el aporte de agua de buena calidad.

Según Agusti (2000) los agrios son sensibles a concentraciones elevadas de sales. Su desarrollo se reduce; las hojas, también de pequeño tamaño, se deshidratan, presentando los primeros síntomas característicamente en sus ápices, y caen.

De acuerdo con Ayers \& Westcot (1987), teniendo como fuente Maas \& Hoffman (1977) y Maas (1984), valores de la conductividad eléctrica del extracto de saturación (CEes) entre $1,7 \mathrm{dS} \mathrm{m}^{-1}$ y $4,8 \mathrm{dS} \mathrm{m}^{-1}$ reduce el rendimiento potencial del cultivo de 100 hasta 50\%, pero el rendimiento potencial es cero cuando la salinidad máxima teórica (CEes) es $8,0 \mathrm{dS} \mathrm{m}^{-1}$, con la cual cesa el crecimiento y desarrollo de la planta. Por su vez, los valores correspondientes para el agua de riego son de 1,1 a $3,2 \mathrm{dS} \mathrm{m}^{-1}$ y $5,3 \mathrm{dS} \mathrm{m}^{-1}$ respectivamente.

Para Agusti (2000) en los suelos salinos la producción de cítricos desciende y los frutos son pequeños, aunque de corteza fina y de coloración más intensa, más dulce y más precoses.

Este trabajo tuvo como objetivo evaluar la variación en la calidad del agua de riego durante el ciclo productivo de un huerto de cítricos y sus consecuencias sobre el suelo y las plantas.
El trabajo se realizó en la temporada 98/99, en la Finca El Saladar, situada a $3 \mathrm{~km}$ al este de la población de Sueca (Valencia), con altitud de $7 \mathrm{~m}$ sobre el nivel del mar, coordenadas geográficas de $39^{\circ} 12^{\prime}$ latitud norte y $0^{\circ} 18^{\prime}$ longitud oeste de Greenwich, la temperatura media anual es de $18,2^{\circ} \mathrm{C}$, y la precipitación y la evapotranspiración media anual son de 564 y de $939 \mathrm{~mm}$, respectivamente. El suelo tiene una textura Arcillosa Fina $\left(8,13 \mathrm{~g} \mathrm{~kg}^{-1}\right.$ de arena, 39,91 $\mathrm{g} \mathrm{kg}^{-1}$ de limo e 51,96 $\mathrm{g} \mathrm{kg}^{-1}$ de arcilla), $\mathrm{pH}=8,57$, conductividad eléctrica del extracto de saturación de 1,09 dS m-1, RAS = 2,51 $\left(\mathrm{mmol}_{\mathrm{C}} \mathrm{L}^{-1}\right)^{1 / 2}$ y CIC = $22,6 \mathrm{cmol}_{\mathrm{C}} \mathrm{kg}^{-1}$. El cultivo era un huerto de cítricos, variedad "Navelate" (Citrus Sinensis (L.) Osbeck), teniendo como patrón "citrange Troyer" (Citrus sinensis (L.) Osbeck x Poncirus trifoliata (L.) Raf), con aproximadamente 11 años de edad, plantado en un marco de $6 \times 4 \mathrm{~m}$, presentando buen estado nutricional. El sistema de riego utilizado es riego localizado con goteros sobre línea, autocompensantes, de $4 \mathrm{~L} \mathrm{~h}^{-1}$, con seis goteros por árbol, tres en cada línea lateral, con goteros agrupados de metro en metro y los agrupamientos separados dos metros entre sí. El sistema de drenaje es subterráneo tipo espina de pescado, con tuberías de cerámica de 6” a cada $6 \mathrm{~m}, 1 \mathrm{~m}$ de profundidad media, descargando en otra tubería también de cerámica de 12 " e de ésta al punto de captación de agua para el riego. El agua de riego es una mezcla no controlada del agua de drenaje con el agua de la Acequia Real del Júcar, esta destinada al cultivo de arroz en la región de la Ribera Baja.

Durante toda la temporada del estudio se hizo un muestreo de las aguas de la A. R. del Júcar, del canal de conducción para el riego de arroz, y la utilizada para el riego de cítricos en la finca, en el sitio destinado a la captación, en las fechas presentadas en la Tabla 1, para determinar su calidad y su variación. También se muestreó en las mismas fechas el agua de drenaje, colectada en pozos de observaciones instalados en cada cuadrante del área del experimento.

Los análisis estadísticos fueron realizados mediante Statgraphics Plus versión 4.0 y de acuerdo con Mate \& Oriol (1996).

El estudio de las relaciones entre variables se ha efectuado descriptivamente mediante diagramas de dispersión y tablas de correlaciones. Sobre la base de esto se han planteado modelos de regresión linear múltiple para predecir algunas variables en función de otras.

\section{RESULTADOS Y DISCUSIÓN}

Los datos respecto a la calidad del agua utilizada en la Finca el Saladar, para el riego en el huerto de naranjo Navelate aparecen en la Tabla 1. Utilizando las directrices establecidas por el Laboratorio de Salinidad de EE.UU (Richards, 1993) y por la FAO (Ayers \& Westcot, 1987) se puede evaluar la calidad de estas aguas y los efectos que el riego con ella puede causar, en el suelo y en los naranjos. 


\section{Salinidad}

Se puede comprobar en la Tabla 1 que hay una variación en la conductividad eléctrica de 1.100 a $3.068 \mu \mathrm{S} \mathrm{cm}^{-1}(1,10$ a 3,07 dS m-1), y por consiguiente una variación en la clase de salinidad de C3 a C4, habiendo aparecido la clase C4 notoriamente en finales de junio inicio de julio, época en la que el riego del arroz se paraliza para realizar los trabajos culturales necesarios. Como el riego del huerto no se interrumpe en este periodo y además la evapotranspiración es muy alta, el agua que está almacenada en las tuberías de drenaje va disminuyendo de cantidad y por tanto va aumentando su concentración de sales, haciendo más peligroso su uso. Lo mismo ocurre desde el mes de septiembre hasta principios de diciembre, final de la temporada de arroz e inicio de la temporada de caza, momento en que se cierra y se abre el paso de agua, respectivamente. En los demás períodos el agua se clasifica como del tipo C3. Por lo tanto, durante la temporada 98/99, el agua fue considerada altamente salina (C3) durante la mayor parte del tiempo, y muy altamente salina (C4) en una determinada época del año. En el primer caso, el agua C3, no puede usarse en suelos cuyo drenaje sea deficiente, y aun usándose con drenaje adecuado se pueden necesitar prácticas especiales de control de la salinidad, debiendo, por lo tanto, seleccionar especies vegetales que sean muy tolerantes a las sales. En el segundo caso (C4), el agua no es apropiada para riego bajo condiciones ordinarias, pero puede usarse ocasionalmente en circunstancias muy especiales. Los suelos deben ser permeables, el drenaje adecuado, debiendo aplicarse un exceso de agua para lograr un buen lavado, y se deben seleccionar cultivos altamente tolerantes a las sales.

Según las directrices de la FAO, los valores de la CE clasifican el agua según su grado de restricción de uso, como ligera a moderada, teniendo solamente un valor (3068 $\mu \mathrm{S} \mathrm{cm} \mathrm{cm}^{-1}$ ), en noviembre (día 190), cuya restricción sería severa.

Valores de conductividad eléctrica del agua de riego superiores a $1,1 \mathrm{dS} \mathrm{m}^{-1}$ ya empiezan a causar problemas para los cítricos (Maas \& Hoffman, 1977; Maas, 1990), aunque los daños por salinidad se intensifican en condiciones de humedad escasa y si el agua de riego tiene niveles moderados de salinidad, por ejemplo, 1,3 dS m (Davies \& Albrigo, 1999), pues determina en gran medida la disponibilidad del agua para la planta a través de su efecto osmótico y consiguiente disminución del potencial total de agua en el suelo. Afortunadamente, las plantas reaccionan aumentando la cantidad de sustancias minerales y orgánicas acumuladas en sus células, lo que produce un aumento de presión osmótica en el interior de la planta, permitiéndoles, de esta manera, mantener el flujo de agua a través del sistema radicular (Pomares, 1986). Ese ajuste osmótico lleva consigo un gasto de energía que se traduce, entre otros efectos, en una disminución del crecimiento vegetativo y de la productividad de las plantas.

\section{Sodicidad}

Utilizando la relación de adsorción del sodio tradicional (RAS) para la clasificación del agua, según las directrices del Laboratorio de Salinidad de EE.UU., se comprueba que el agua se mantiene durante toda la temporada con una concentración baja en sodio (S1), pudiendo ser usada en la mayoría de los suelos con poca probabilidad de alcanzar niveles peligrosos de sodio intercambiable. Al evaluar las condiciuones salinas tomando como base el RAS ${ }^{\circ}$ (RAS corregido), en los mismos periodos en que el agua cambia de clase de salinidad (C3 a C4), la sodicidad cambia de S1 a S2, que

Tabla 1. Valores medios de los análisis químicos de los elementos del agua de riego utilizada en el ensayo, en la temporada 98/99.

\begin{tabular}{|c|c|c|c|c|c|c|c|c|c|c|c|c|c|c|c|c|}
\hline \multirow{2}{*}{$\begin{array}{l}\text { Fecha } \\
\text { (Día) }\end{array}$} & \multirow{2}{*}{$\begin{array}{c}\text { CE } \\
\mu \mathrm{S} \mathrm{cm}^{-1}\end{array}$} & RAS & $\mathrm{RAS}^{\circ}$ & \multicolumn{2}{|c|}{ Clase } & \multirow{2}{*}{ pH } & \multirow{2}{*}{$\begin{array}{l}\text { PSI } \\
\%\end{array}$} & \multirow{2}{*}{$\begin{array}{c}\mathrm{TSD} \\
\mathrm{mg} \mathrm{L} \mathrm{L}^{-1}\end{array}$} & \multicolumn{5}{|c|}{ Cationes meq $\mathrm{L}^{-1}$} & \multicolumn{3}{|c|}{ Aniones meq $\mathrm{L}^{-1}$} \\
\hline & & $\left(\mathrm{mmol}_{\mathrm{c}}\right.$ & $\left.L^{-1}\right)^{1 / 2}$ & (1) & (2) & & & & $\mathrm{Ca}^{++}$ & $\mathrm{Mg}^{++}$ & $\mathrm{Na}^{++}$ & $\mathbf{K}^{+}$ & SumaC & $\mathrm{Cl}^{-}$ & $\mathrm{SO}_{4}=$ & $\mathrm{CO}_{3} \mathrm{H}$ \\
\hline $5 / 5(1)$ & 1225 & 1,44 & 1,73 & C3S1 & C3S1 & 8,49 & 23,8 & 784.0 & 6.80 & 3.60 & 3.29 & 0.14 & 13.83 & 4.23 & 6.64 & 2.96 \\
\hline $21 / 5(20)$ & 2088 & 4,38 & 4,79 & C3S1 & C3S2 & 8,27 & 47,3 & 1336.3 & 5.20 & 6.40 & 10.56 & 0.14 & 22.32 & 7.89 & 11.47 & 2.96 \\
\hline $20 / 6(50)$ & 1416 & 2,27 & 2,76 & C3S1 & C3S1 & 8,70 & 33,0 & 906.2 & 6.00 & 4.40 & 5.18 & 0.12 & 15.70 & 3.94 & 7.32 & 4.44 \\
\hline $23 / 6(53)$ & 2364 & 4,33 & 4,44 & C4S1 & C4S2 & 8,27 & 45,0 & 1513.0 & 6.00 & 7.60 & 11.29 & 0.19 & 25.08 & 10.42 & 12.97 & 1.69 \\
\hline $3 / 7(63)$ & 2568 & 3,66 & 4,54 & C4S1 & C4S2 & 8,70 & 39,2 & 1643.5 & 9.20 & 6.40 & 10.21 & 0.20 & 26.01 & 8.17 & 12.66 & 5.18 \\
\hline $7 / 7(67)$ & 1562 & 2,29 & 2,43 & C3S1 & C3S1 & 8,38 & 32,4 & 999.7 & 5.20 & 6.00 & 5.43 & 0.14 & 16.77 & 5.63 & 9.03 & 2.11 \\
\hline $14 / 7(74)$ & 1209 & 1,44 & 1,52 & C3S1 & C3S1 & 8,26 & 22,9 & 773.8 & 4.40 & 7.20 & 3.47 & 0.11 & 15.18 & 3.94 & 9.13 & 2.11 \\
\hline $7 / 8(97)$ & 1316 & 2,24 & 2,29 & C3S1 & C3S1 & 8,25 & 32,9 & 842,2 & 4,40 & 5,60 & 5,01 & 0,23 & 15,24 & 2,82 & 10,73 & 1,69 \\
\hline 21/8 (111) & 1211 & 1,91 & 2,13 & C3S1 & C3S1 & 8,28 & 31,7 & 775,0 & 4,80 & 4,00 & 4,13 & 0,11 & 13,04 & 2,82 & 8,00 & 2,22 \\
\hline 25/8 (115) & 1100 & 1,63 & 1,72 & C3S1 & C3S1 & 8,27 & 27,2 & 704,0 & 4,40 & 4,00 & 3,34 & 0,52 & 12,26 & 2,82 & 7,33 & 2,11 \\
\hline $31 / 8$ (121) & 2175 & 3,10 & 3,72 & C3S1 & C3S1 & 7,75 & 35,1 & 1392,0 & 8,40 & 7,60 & 8,78 & 0,20 & 24,98 & 5,63 & 14,49 & 4,86 \\
\hline $15 / 9(136)$ & 2013 & 3,23 & 3,51 & C3S1 & C3S1 & 8,24 & 38,8 & 1288,3 & 6,40 & 6,40 & 8,18 & 0,12 & 21,10 & 7,61 & 11,38 & 2,11 \\
\hline $29 / 9(150)$ & 2568 & 4,46 & 4,79 & $\mathrm{C} 4 \mathrm{~S} 1$ & C4S2 & 8,25 & 44,1 & 1643,5 & 7,20 & 7,60 & 12,14 & 0,56 & 27,50 & 12,39 & 13,00 & 2,11 \\
\hline 11/11 (193) & 3068 & 3,66 & 4,81 & C4S1 & C4S2 & 7,77 & 32,2 & 1963,5 & 13,60 & 8,40 & 12,14 & 0,30 & 34,44 & 10,42 & 18,01 & 5,71 \\
\hline $17 / 11(200)$ & 1756 & 1,79 & 2,29 & C3S1 & C3S1 & 8,64 & 24,0 & 1123,8 & 9,60 & 6,00 & 5,01 & 0,23 & 20,84 & 4,79 & 11,08 & 4,91 \\
\hline Media & 1843 & 2,79 & 3,16 & & & 8,30 & 34,0 & 1179,3 & 6,77 & 6,08 & 7,21 & 0,22 & 20,29 & 6,23 & 10,88 & 3,14 \\
\hline
\end{tabular}

(1) - Clasificación del agua con base en el RAS tradicional; (2) - Clasificación del agua con base en el RAS ${ }^{\circ}$ corregido; PSI - Porcentaje de sodio intercambiable (PSI = Na/Suma C) 
caracteriza un agua media en sodio y que en suelos de textura fina, como él del área de ensayo, representa un peligro considerable, más aún cuando dicho suelo posee una alta CIC (capacidad de intercambio de cationes), especialmente bajo condiciones de lavado deficiente, a menos que el suelo contenga yeso.

Usando los valores del RAS $^{\circ}$ que varían entre 1,52 y $4,81\left(\mathrm{mmol}_{\mathrm{C}} \mathrm{L}^{-1}\right)^{1 / 2}$ y los valores de la CEar que varían de 1,1 a $3,07 \mathrm{dS} \mathrm{m}^{-1}$ (es decir mayor que $0,7 \mathrm{dS} \mathrm{m}^{-1}$ ), y utilizando las directrices de la FAO, se puede afirmar que el agua no presenta ningún grado de restricción de uso

En la Figura 1 se observa el comportamiento de las variables RAS (tradicional), RAS $^{\circ}$ (corregido) y CEar (conductividad eléctrica del agua de riego) durante la temporada 98/99. Se puede ver que las curvas tienen la misma tendencia, exceptuando los puntos referentes a los 63 y 193 días cuando las curvas de RAS $^{\circ}$ y CEar suben y la curva de RAS tiende a bajar. En estos puntos el valor de la concentración de calcio en la interfaz suelo-agua, $\mathrm{Ca}^{\circ}$, es mucho menor que la concentración de calcio en el agua, $\mathrm{Ca}$, indicando que hubo una gran precipitación de calcio. Se comprueba también que el $\mathrm{RAS}^{\circ}$ es mayor o igual al RAS y que la curva de la CEar es semejante a la curva del RAS ${ }^{\circ}$.

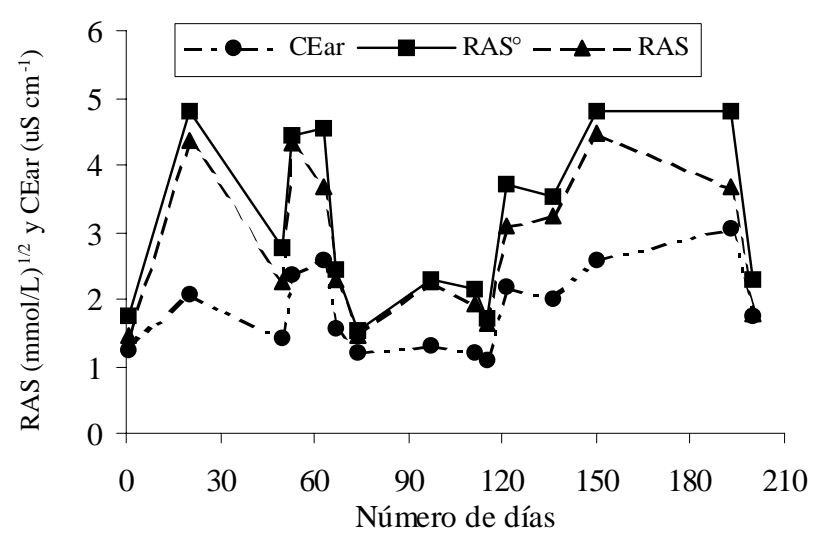

Figura 1. Comportamiento del RAS ${ }^{\circ}$, RAS y de la CEar del agua de riego en el área del ensayo, en la temporada 98/99

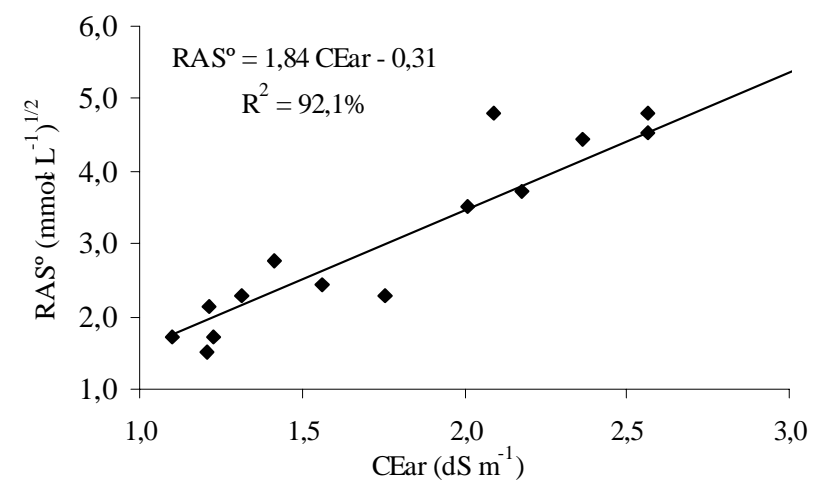

Figura 2. Relación entra la conductividad eléctrica del agua de riego e el relación de adsorción de sodio corregido

\section{Relación RAS ${ }^{\circ}$ - Conductividad Eléctrica}

La relación RAS $^{\circ}$ y CEar se ajusta a un modelo lineal definido por la siguiente recta (Figura 2):

La pendiente 1,84, indica el incremento de la variable RAS $^{\circ}$ para un incremento de una unidad en la variable CEar. Es decir, para cada $\left(\mathrm{dS} \mathrm{m}^{-1}\right)$ adicional en el CEar, el RAS ${ }^{\circ}$ aumentará aproximadamente 1,84 $\left(\mathrm{mmol} \mathrm{L}^{-1}\right)^{1 / 2}$.

\section{Relación RAS - Conductividad Eléctrica}

Era de esperar que la relación mas fuerte entre RAS y CEar fuese también lineal. Pero, la mejor representación de la relación entre las dos variables ha sido el modelo potencial $\mathrm{Y}=\mathrm{a}^{*} \mathrm{X}^{\mathrm{b}}$ definido por la siguiente curva (Figura 3):

La diferencia de un 17,5\% entre los coeficientes de determinación del RAS ${ }^{\circ}$ y del RAS, ambos en relación con la CEar, se puede afirmar que la estimativa de la sodicidad en función de la $\mathrm{RAS}^{\circ}$ es más razonable que mediante la RAS.

\section{Carbonato de sódio residual - CSR}

Utilizando la ecuación $\mathrm{CSR}=\left(\mathrm{CO}_{3}{ }^{=}+\mathrm{HCO}_{3}\right)-\left(\mathrm{Ca}^{++}+\mathrm{Mg}^{++}\right)$ y los datos de la Tabla 1 , se verifica que los valores del CSR son siempre menores que cero, indicando que el agua utilizada durante toda la temporada fue probablemente apta para riego, sin provocar problemas de sodicidad en el suelo.

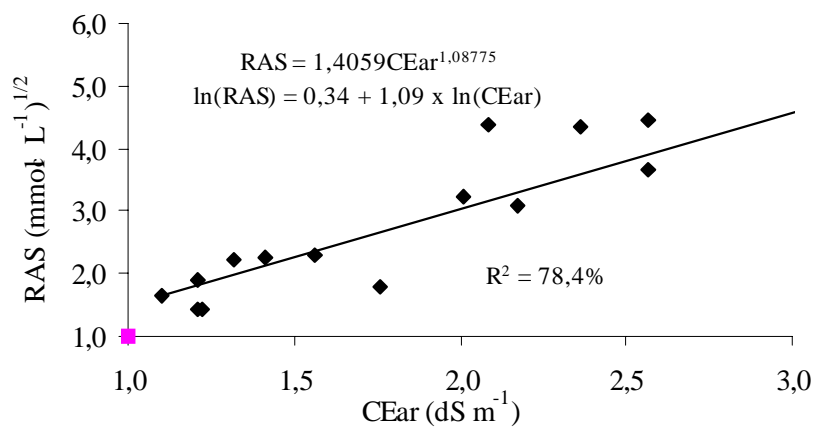

Figura 3. Relación entre la CEar e el RAS del agua de riego utilizada

\section{Toxicidad por sodio o cloruro.}

Para valores de $\mathrm{RAS}^{\circ}$ menores de 3 y la concentración de sodio menor de 3 meq $\mathrm{L}^{-1}$ no hay restricción para riego por superficie o por aspersión respectivamente (Ayers \& Westcot, 1987). En la Tabla 1 se puede observar que la RAS $^{\circ}$ varió de 1,52 a 4,81 teniendo como media $3,16\left(\mathrm{mmol} \mathrm{L}^{-1}\right)^{1 / 2}$, indicando esta variación un paso de ninguna restricción a ligera a moderada restricción para su uso en riego por superficie. Los valores de las concentraciones de $\mathrm{Na}$ son siempre superiores a 3 meq $\mathrm{L}^{-1}$ clasificando al agua con ligera a moderada restricción de uso para riego por aspersión. Sin embargo, Gómez Lucas \& Pedreño (1992), citando Cerdá et al., afirmaron que concentraciones de $\mathrm{Na}<0,25 \mathrm{~g} \mathrm{~L}^{-1}$

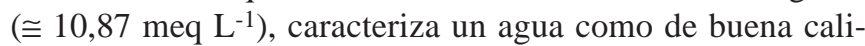
dad y solamente se considera de mala calidad cuando su con-

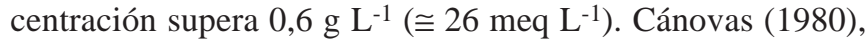
considera que toda agua con concentración de $\mathrm{Na}>0,3 \mathrm{~g} \mathrm{~L}^{-1}$

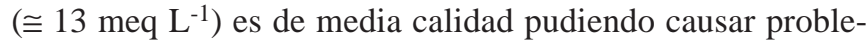
mas para las plantas y suelos. En esas condiciones, el agua 
en la mayor parte del tiempo se considera de buena calidad, salvo cuando los valores de $\mathrm{Na}>10 \mathrm{meq} \mathrm{L}^{-1} \mathrm{y}$, por coincidencia, la sodicidad se clasifica como S2, el agua se considera de calidad media.

Como los valores del porcentaje de sodio intercambiable (PSI), oscilan entre 22,9 y 47,3\%, clasifica el agua como solamente utilizable para cultivos semitolerantes o tolerantes, con restricción de uso para naranjo (Citrus sinensis) por ser sensible a valores de PSI superiores al 15\% (Pearson, 1960; Ayers \& Westcot, 1987, FAO-UNESCO, 1973).

Las concentraciones de cloruros oscilan entre 2,82 y 12,39 meq $\mathrm{L}^{-1}$ (Tabla 1), ocurriendo los valores superiores a 10 meq $\mathrm{L}^{-1}$ cuando la clasificación de salinidad es C4, caracterizando la influencia del cloruro en la conductividad eléctrica del agua.

Para Ayers \& Westcot (1987) las aguas con concentraciones de cloruros entre 4 y 10 meq $\mathrm{L}^{-1}$ tienen restricción ligera a moderada y cuando las concentraciones son superiores a 10 meq $\mathrm{L}^{-1}$ poseen una severa restricción para riego por superficie. En cuanto al riego por aspersión, las aguas con concentraciones de cloruros mayores que 3 meq $\mathrm{L}^{-1}$, poseen ya severas restricciones.

Específicamente, para el porta injerto Citrange troyer el valor máximo permisible en riego por superficie es de 6,7 meq $\mathrm{L}^{-1}$. Por tanto, durante la temporada el agua varió de grado de ninguna restricción a severa, ocurriendo este último en periodos muy pequeños del periodo crítico de riego (finales de junio inicio de julio), o cuando el riego no era tan necesario para el desarrollo del cultivo (finales de septiembre a inicio de noviembre). Aún citando Cerdá et al., Gómez Lucas \& Pedreño (1992), consideran que el agua es de buena calidad cuando las concentraciones de cloruros son inferiores a $0,30 \mathrm{~g} \mathrm{~L}^{-1}\left(\cong 8,5 \mathrm{meq} \mathrm{L}^{-1}\right)$ y solamente se considera de mala calidad con concentraciones superiores a 0,7 g L $\mathrm{g}^{-1}\left(\cong 19,7 \mathrm{meq} \mathrm{L} \mathrm{L}^{-1}\right.$ ). Cánovas (1980) considera el agua de calidad media aquella que posee concentraciones de cloruros superiores a $0,5 \mathrm{~g} \mathrm{~L}^{-1}\left(\cong 14,1 \mathrm{meq} \mathrm{L}^{-1}\right)$

\section{pH}

El pH del agua varió de 7,75 a 8,70, prácticamente en el rango de valores considerados normales por Ayers \& Westcot (1987), que es de 6,5 a 8,4. Una de las consecuencias del valor alto del $\mathrm{pH}$ es la destrucción de las raíces y la reducción de la asimilación de fosfatos, hierro, cinc, cobre y manganeso (Herendia, 1999). También puede crear problemas de obstrucción en los sistemas de riego localizado (Nakayama, 1982) y, por supuesto, reducción en la eficiencia de aplicación de agua y fertilizantes, provocando la pérdida de uniformidad del huerto y de los frutos y una cosecha deficitaria.

\section{CONCLUSIONES}

1. La mezcla de las aguas de drenaje con el agua de la Acequia Real del Júcar, según las directrices de la FAO, no presenta ningún grado de restricción de uso;

2. En las condiciones en que se realizó los ensayos, es posible la utilización de la mezcla de agua de buena calidad con una agua de calidad dudosa durante un largo periodo de tiempo, sin salinizar ni sodificar el suelo;

\section{LITERATURA CITADA}

Agusti, M. Citricultura. 1.ed. Madrid: Ediciones Mundi-Prensa, 2000 416p.

Ayars, J. E.; Hutmacher, R. B.; Schoneman, R. A.; Vail, S. S. Trickle irrigation of sugar beets with saline drainage water. Annual Report of the Water Management Research Laboratory USDA-ARS, Fresno, California, 1986a. p.5-6.

Ayars, J. E.; Hutmacher, R. B.; Schoneman, R. A.; Vail, S. S.; Felleke, D. Drip irrigation of cotton with saline drainage water. Transactions of the ASAE v.29, n.6, p.1668-1673, 1986b.

Ayers, R. S.; Westcot, D. W. La calidad del agua para agricultura (Estudios FAO: Riegos y Drenajes $n^{0} 29$. Roma: Re. FAO. 1987. 174p.

Canovas, J. Calidad agronómica de las aguas de riego. Publicaciones Extensión Agraria. Madrid. 1980. 55p.

Daveis, F. S.; Albrigo, L. G. Cítricos: F. S. Daveis y L.G. Albrigo. Zaragoza: Editorial ACRIBIAS S.A. 1999. 283p.

FAO/UNESCO, Irrigation, drainage and salinity. An international sourcebook. Paris: UNESCO/Hutchinson, 1973. 510p.

Gómez Lucas, N.; Pedreño, M. B. Aguas de riego: Análisis e interpretación. 1.ed. Alicante: Secretariado de publicaciones. Universidad de Alicante, 1992. 63p.

Grattan, S. R. Irrigation with saline water. In: K. K. Tanji; B. Yaron (eds): Management of water use in agriculture. SpringerVeriag Berlin Heidelberg New York. New York: Advanced Series Agricultural Science - ASAS, v.22. 1994. Chap. 8, p.179-198.

Grattan, S. R.; Shennan, C.; May, D. M.; Mitchel, J. P. Burau, R. $\mathrm{G}$. Use of drainage water for irrigation of melons and tomatoes. California Agricultural. v.41, n.9, p. 27-28. 1987

Grattan, S. R.; Rhoades, J. D. Irrigation with saline ground water and drainage water. In: K. K. Tanji (es). Agricultural Salinity Assessment and Management. Manuals and Reports on Engineering Practice n.71, New York: America Society of Civil Engineers - ASCE, 1990. Chap. 20, p.432-449.

Herendia, E. La salinidad en los árboles frutales. Nueva propuesta de clasificación de suelos y aguas en función de la salinidad. Castelldefels - Barcelona: Fruticultura Profesional, n.107, p.19-30. 1999.

Howitt, R. E.; M’Marete, M. Well set aside proposal: a scenario for ground water banking. California Agriculture, v.45, n.3, p.69. 1991.

Maas, E. V. Salt tolerance of plants. In: Christie, B. R. (ed.) The handbook of plant science in agriculture. Boca Raton: CRC Press, 1984.

Maas, E. V. Crop salt tolerance. In: K. K. Tanji (ed): Agricultural Salinity Assessment and Management. Manuals and Reports on Engineering Practice, 71, New York: America Society of Civil Engineers - ASCE, 1990. Chap. 13, p.262-304.

Maas, E. V.; Hoffman, G. J. Crop salt tolerance-current assessment. Journal of Irrigation Drainage Division of ASCE, v.103, n.IR2: p.115-134. 1977. 
Mate, C.; Oriol, N. Curso general sobre Statgraphics. Anexo para la versión plus for Windows. 1.ed. Madrid: Universidad Pontificia de Comillas - ICAI-ICADE. 1996. 209p.

Meiri, A.; Shalhevet, J. Stozi, L. H.; Sinai, G.; Steinhardt, R. Managing multi-source irrigation water of different salinities for optimum crop production. BARD Tech Rep 1-402-81. Volcanic Center, Bet Dragon, 1986, 172p.

Nakayama, F. S. Water analysis and treatment techniques to control emitter plugging. In: Process Irrigation Association Conference, Oregon: Portland, 1982.

Pearson, G. A. Tolerance of crop to exchangeable sodium. Washington DC, USDA, 1960. 4p. Information Bulletin, 216.

Pomares, F. La salinidad del suelo en los cítricos. Generalidad valenciana - Conselleria d'agricultura, pesca y alimentación. Generalitat valenciana, Valencia: CAPA, 1986. 24p.

Rhoades, J. D. Quality of water for irrigation. Soil Science U.S.A., v.113, p.277-284. 1972.

Rhoades, J. D. Potential for using saline agricultural drainage waters for irrigation. In: Process Water management for irrigation and drainage. Reno: ASCE. 1977. p.85-116.
Rhoades, J. D. Use of saline water for irrigation. California Agriculture, Oak Ridge, v.38, n.10, p.42-43. 1984.

Rhoades, J. D., Binghan, F. F.; Letey, J.; Dedrick, A. R.; Bean, M.; Hoffman, G. J.; Alves, A. W.; Swain, R. V.; Pacheco, P. G.; Le Mert, R. D. Reuse of drainage water for irrigation: results of Imperial Valley study. I. Hypothesis, experimental procedure and cropping results. Higardia, Oakland, v.56, n.5, p.1-16. 1988.

Rhoades, J. D., Binghan, F. F.; Letey, J.; Hoffman, G. J.; Dedrick, A. R.; Pinter, P. J. Replogle, J. A. Use of saline drainage water for irrigation: Imperial Valley Study. Agricultural Water Management, Amsterdam, v.16, p.25-36. 1989.

Richards, L. A. Diagnóstico y recuperación de suelos salinos y sódicos. Personal de Laboratorio de Salinidad de EE.UU. 6.ed. 7. reimpresión. México: Editorial LIMUSA. 1993. 176p.

Shennan, C.; Grattan, S.; May, D.; Burau, R.; Hanson, B. Potential for the long-term cyclic use of saline drainage water for the production of vegetable crop. Technical Progress Report, U.C. Salinity/Drainage Task Force, Division of Agriculture and Natural Resource, University of California, Davis, p.142146. 1987. 